\title{
Liderazgo y emprendimiento: ¿cuál es su relación?
}

\author{
Marlon Santiago Leal Paredes \\ msleal@utn.edu.ec \\ Universidad Técnica del Norte \\ Ibarra - Ecuador \\ Carla Susana Orozco Espinosa \\ corozco@institutocotacachi.edu.ec \\ Instituto Superior Tecnológico Cotacachi \\ Cotacachi - Ecuador \\ Edwin Iván Guamán Mullo \\ eguaman@institutocotacachi.edu.ec \\ Instituto Superior Tecnológico Cotacachi \\ Cotacachi - Ecuador
}

\section{RESUMEN}

El presente artículo tiene como objetivo presentar varias reflexiones sobre la posible relación entre el liderazgo y el emprendimiento, para lo cual se realiza una búsqueda de información en las distintas bases de datos académicas y científicas, que se encuentran en la red, sostenidas en una estrategia metodológica para describir y explicar el comportamiento de estos dos elementos y su interacción en el ámbito del liderazgo y la generación de ideas de negocios, basándose en artículos científicos de prestigiosas revistas, para lo cual se aplicó métodos, procesos y técnicas para la recolección de información, con énfasis en la revisión de literatura, análisis sintético y observación. Como resultado se pudo dar contestación a la interrogante de la posible relación de estas dos variables, obteniendo una amplia reflexión y explicación sobre la temática planteada que será beneficiosa para los investigadores al momento de estudiar estos componentes.

Palabras clave: liderazgo; emprendimiento; organizaciones. 


\title{
Leadership and entrepeneurship: what is their relationship?
}

\begin{abstract}
This article aims to present various reflections on the possible relationship between leadership and entrepreneurship, for which a search of information was carried out in the different academic and scientific databases that are available on the web, supported by a methodological strategy to describe and explain the behavior of these two elements and their interaction in the field of leadership and the generation of business ideas. Considering scientific articles from prestigious magazines, applying their methods, processes, and techniques to collect information, with emphasis on literature review, synthetic analysis, and observation. As a result, it was possible to answer the question of the possible relationship between these two variables, obtaining a broad reflection and explanation on the issue raised that will be beneficial for researchers when studying these components.
\end{abstract}

Keywords: leadership; entrepreneurship; organizations.

Artículo recibido: 15 noviembre. 2021 Aceptado para publicación: 10 diciembre 2021 Correspondencia: msleal@utn.edu.ec Conflictos de Interés: Ninguna que declarar 


\section{INTRODUCCIÓN}

El propósito de la investigación busca aportar una reflexión sobre la relevancia que posee el liderazgo en la generación de emprendimientos, dar la contestación a la pregunta planteada sobre ¿Cuál es su relación?, esto con la finalidad de contribuir a la literatura con un análisis sobre el comportamiento de estas dos variables y como su posible relación tiene efectos en el éxito de los emprendimientos. En referencia al método de investigación para elaborar la reflexión sobre el tema abordar, se utilizó un enfoque cualitativo, usando la técnica de revisión ordenada, la que permite desarrollar una síntesis de los artículos y autores de un tema en particular, esta característica permite enlazar los resultados que se obtengan, para posteriormente ser comunicados en relación a un análisis narrativo o descriptivo.

Cuando las personas describen negocios exitosos, siempre se hace referencia al gerente o al fundador, identificándose como un profesional o individuo con características muy particulares que habitualmente el resto de personas no posee. Al indagar de manera más profunda, una de las características que sobresalen es el Liderazgo, usualmente se asocia a personas triunfadoras que han logrado alcanzar todos objetivos socialmente deseables. Generando se desarrollen estudios enfocados alrededor de la temática del liderazgo, permitiendo que a través de las investigaciones se promueva un campo de estudio donde se indaga y explora los componentes y elementos que conforman este tópico con la finalidad de examinar sus alcances y cómo este se relaciona e influye con otras actividades y áreas de estudio.

Es así como se han promovido decenas de investigaciones para entender el impacto que el liderazgo tiene en las organizaciones a través de las habilidades blandas que ejerce el líder con sus colaboradores, identificando los factores que ayuden a que exista una sinergia en los procesos internos y externos de los negocios para cumplir con los objetivos planteados (Leal, 2020).

Esto ha llevado a que se analice los aspectos más relevantes de esta temática y considerar cómo afecta a otras actividades como por ejemplo el espíritu emprendedor (Reid et al., 2018). En este sentido Aiyappa y Mahesh (2020), indican que, el liderazgo si tiene estrecha relación con el emprendimiento, pues se han encontrado evidencias que las personas emprendedoras gozan de ciertas similitudes con las personas definidas como líderes siendo estas: una visión a medio y largo plazo, búsqueda de la cristalización de 
objetivos, tenacidad, valores éticos y transparencia. Lo que permite entender que estas características benefician a que los pequeños negocios se vuelven exitosos en el tiempo. Según Galloway, Kapasi, y Sang (2015) el emprendedor es una persona con capacidades creativas e innovadoras, permitiéndole identificar oportunidades de negocio en nichos que usualmente no pueden ser considerados por otras personas, además, de ser un visionario, apasionado, determinado y persistente con el logro de los objetivos. Dando a denotar su capacidad de líder lo que conlleva a que las personas confíen plenamente en sus dotes y cualidades.

Con base a lo anterior es que nace el término Líder Emprendedor, siendo aquel personaje que ostenta las características de estos dos elementos, permitiendo facilidades al momento de generar ideas y al mismo tiempo motivar a los socios o colaboradores a que crean en sus palabras al instante de proponer ideas de negocio. En ese sentido Lewis (2015) manifestó que las iniciativas obtienen un valor agregado, observando que muchas personas que desarrollan sus negocios los terminan vendiendo a inversionistas, pero simultáneamente los contratan como directores o gerentes de su mismo emprendimiento, por la confianza que brindan hacia los accionistas, ya que ellos conocen el potencial de su negocio.

Es así que a través del tiempo se ha estudiado estas dos variables por separado, pero en la actualidad se denota que la estrecha relación que tiene el liderazgo con el emprendimiento termina siendo obvio, pero las reflexiones de cómo estas se involucran mutuamente sigue siendo desconocida por muchas personas. Por tal razón esto permite a los investigadores desarrollar propuestas de nuevas investigaciones para explicar su correlación, dando así respuesta a diversas interrogantes planteadas.

\section{DESARROLLO}

El liderazgo es definido como las habilidades que posee una persona para influenciar sobre un grupo de trabajo, para direccionarlos en la consecución de metas u objetivos (Leal, et al., 2021). Por otra parte, se comprende que el emprendimiento es el proceso de diseñar y administrar un nuevo negocio, que habitualmente suele comenzar con una idea donde se ofertan productos o servicios al público (Chan et al., 2012). Varios estudios relacionan estos dos campos de conocimientos dentro del giro de los negocios, ya que por un lado se resalta las capacidades de una persona para direccionar a un grupo, también se 
hablan de las capacidades de los individuos para generar ideas de negocios, siendo estas características de un visionario (Vecchio, 2003).

El liderazgo, es una temática de amplia coyuntura, pues se involucra en distintos giros de negocios en los cuales permite su mejora o declive en la dirección de las organizaciones (Sarabi, Froese, Chng, \& Meyer, 2020), eso incluye a los emprendimientos los cuales tienen un gran impacto dentro de la economía y al mismo tiempo son fuentes de generación de empleo (Aiyappa \& Mahesh, 2020). Es así que el análisis del comportamiento del líder y su relación con el emprendimiento son importantes dentro del entorno empresarial y comprender cómo estos elementos permiten generar un desarrollo sostenible para los negocios, lo vuelve atractivo como fuente de estudio y reflexión (Santos, Steil, \& Delgado-Hernández, 2020).

En la actualidad el liderazgo dentro de los negocios se vuelve cada vez un término más común en el desempeño de las organizaciones, pero de la misma forma los distintos estilos de liderazgo están enfocados a determinados segmentos o sectores empresariales, surgiendo una expresión que actualmente es conocida como el Liderazgo Emprendedor, siendo este un elemento de innovación e implementación de innovaciones vanguardistas en la concepción de ideas que se acoplan a los entornos, asumiendo riesgos y enfrentando los retos que representan los mercados competitivos, alineándose con el emprendimiento estratégico para buscar las mejores opciones para abrirse camino y consolidarse en un nicho de mercado (Subramaniam \& Shankar, 2020)

En el mismo contexto Aguinis et al. (2020), manifestaron que el liderazgo y el emprendimiento tienen una relación en su impacto en las investigaciones que se realizan a nivel mundial, y significativamente en la actualidad al ser tópicos que generan debate en la comunidad científica al estudiar su importancia dentro de la empresa y cómo estos se vuelven elementos a considerar para los campos de adiestramiento en los nuevos gerentes, quienes deben tener una visión más amplia de la realidad, y como las actitudes e iniciativas van ejercer una influencia dentro de su ámbito laboral.

Por otra parte, Zbierowski (2020), expresó que no se puede generalizar el liderazgo para todos los tipos de organización, en virtud de que las características de los individuos influyen de varias formas a la empresas, pues cada una posee una cultura a la cual se debe adaptar el líder, estas implicaciones sugieren que no todos los líderes son capaces de afrontar los retos que ebulle a los emprendimientos y los desafíos que estos suponen al 
tener que ser creativos en sus giros de negocio para mantenerse y lograr alcanzar los objetivos. Considerando que la motivación que posee las personas suelen ser los motores que influyen en la iniciación de los emprendimientos, estos motores pueden ser de índole económico, social y familiar, pero no cabe solo con tener una motivación de superación, además, se debe complementar con una visión y liderazgo con un enfoque estratégico y flexible (Chan et al., 2012).

De la misma forma Chang, Chang, y Chen (2020) investigaron la relación que existe entre el liderazgo y el emprendiendo, en su estudio encontraron que estas dos variables conservan una relación que beneficia a los nuevos negocios que se encuentran en fase de desarrollo, lo que permite que puedan crecer y expandirse siguiendo la visión del líder, la cual se afianza en su ímpetu de progresar y concretar los objetivos que idealizo. Siguiendo la misma línea Cogliser y Brigham (2004) expresaron que el liderazgo y el emprendimiento son constructos que se han estudiado por separado hace varios años, pero los estudios y análisis respecto a su relación siguen siendo motivo de nuevas propuestas de investigaciones que ayudan a comprender cómo estos dos puntos se interrelacionan. La visión del liderazgo y el emprendimiento, no termina ciertamente tan descontextualizada, sino por lo contrario se vuelve un campo atractivo para el desarrollo de ideas donde se puede indagar cómo el liderazgo afecta a los emprendimientos, pues a pesar de que estos surgen de una motivación o necesidad, no siempre terminan siendo exitosos en el tiempo, quizás puede ser por la falta de creatividad, falta de innovación o simplemente falta de visión del emprendedor, pero estudios demuestran que un factor determinante yace en la capacidad del líder (Reid et al.,2018). Además, no solamente el liderazgo debe ser visto desde la perspectiva del fundador como tal, sino de la persona que tiene la capacidad para liderar un grupo de emprendimientos y buscar unificar los mismo para que se vuelvan emprendimientos corporativos, ya que al poseer esta capacidad este individuo puede proyectar los negocios a otra escala y generar un beneficio colectivo (Boukamcha, 2019).

Para Vecchio (2003), el pensar que el emprendimiento es un área de conocimiento diferente a otros dominios como el liderazgo, es una idea errada, pues se ha demostrado en estudios que tiene una relación más cercana de lo que muchos podrían pensar, en vista que comparten varios elementos como por ejemplo: aspectos personales, formación y hábitos, siendo estos parámetros de comparación de las características de los individuos 
que poseen una visión de superación y que buscan objetivos alcanzables. Como se demuestra a través del estudio realizado por Morakinyo y Akinsola (2019), en el que se evidencio que la instrucción en estas dos temáticas contribuye significativamente a la generación de iniciativas en el sector productivo al proponer ideas de negocios que sean factibles en el territorio.

El contexto y la cultura de los países es un factor importante en la generación de emprendimientos en cada uno de ellos, las oportunidades que se brindan a través de las políticas públicas y lideradas por el Estado, permite que se canalicen recursos para impulsar estos negocios, pero estas políticas no solo deben enfocarse en la parte económica, pues es necesario también el desarrollo del individuo y sus capacidades de liderazgo (Leal et al., 2021). En la misma idea Stephan y Pathak (2016), ratificaron que la cultura influye sustancialmente en los individuos al momento de referirse al liderazgo y al emprendimiento, pues estos elementos se van descubriendo en las distintas etapas de la vida y se forjan con el pasar de los años, donde estas características maduran y logran afianzarse.

El liderazgo no necesariamente debe estar ligado a la masculinidad, ya que se ha identificado que las mujeres son capaces de generar emprendimientos más efectivos en función de sus habilidades y su facilidad en la adaptación de actividades y multiactividades simultáneas que pueden desempeñar y beneficiar a la concepción de iniciativas emprendedoras (Galloway, Kapasi, \& Sang, 2015). Permitiendo que las mujeres con el pasar del tiempo afiancen sus conocimientos en el liderazgo emprendedor, y logrando promulgar esta actividad entre sus pares, liderando y beneficiando con estas actividades el desarrollo en los emprendimientos de las mujeres, para que se consoliden y generen rentabilidad (Lewis, 2015).

Se debe comprender que los emprendimientos inicialmente nacen desde una visión de superación personal por un individuo, siendo en su mayoría de veces inconsciente de los riesgos que puede correr al fomentar una actividad sin el más mínimo conocimiento del área de incursión, por tal razón la mayoría de negocios comienzan en la informalidad, sin un rumbo concreto de destino, teniendo varias inseguridades de si se tendrá éxito o será un fracaso a mediano plazo. Pero a pesar de estas problemáticas, las cualidades que posee el emprendedor son fundamentales, pues éstas permitirán que sobresalga sus habilidades de liderazgo y proyección que ayuden a que el emprendimiento pueda desarrollarse y 
logre sobrevivir a pesar de las distintas adversidades que se presenten en el camino (Morakinyo \& Akinsola, 2019; Leal, 2020; Subramaniam \& Shankar, 2020).

\section{CONCLUSIÓN O CONSIDERACIONES FINALES}

El estudio y el conocimiento sobre el liderazgo y su relación con el emprendimiento, sigue siendo un tema que manifiesta varias dudas alrededor del entorno académico, pero brindan pautas de poder relacionar estos campos de conocimiento y su implicación dentro de los negocios y más aún si se habla alrededor del comportamiento de los individuos que emprenden.

Pero se observa que su relación va más allá de las similitudes que poseen entre sí, sino más bien en cómo las personas cultivan estas capacidades y las desarrollan en el tiempo, a través de sus experiencias y aprendizajes a lo largo de su vida, permitiéndoles solidificar su aportación al crecimiento de los negocios.

La falta de políticas públicas que ayuden al crecimiento de los emprendimientos sigue siendo escasas, pues a pesar de las personas que inicien su negocio y su latente temor de saber hasta cuando funciona, es donde la aplicación y el direccionamiento correcto de fondos para instrucción de estos visionarios es fundamental, pues permitirá consolidar sus objetivos de una manera más clara y realista, además, de generar su desarrollo personal aflorando sus cualidades como líder.

Sin duda alguna, el líder emprendedor es un ideal que debe buscar cada individuo e identificar internamente estas capacidades que lo ayude a sobrellevar los riesgos a futuro que tendrá al momento de comenzar su negocio. Como se ha venido analizando en la literatura, nos lleva a la reflexión y conclusión de que hay varios componentes que conforman un emprendedor exitoso, y uno de los más relevantes es el liderazgo, es así como se define que el liderazgo tiene una estrecha relación con el emprendimiento, manifestándose de distintas formas en cada persona y como al final tiene su impacto en las personas, sociedades y organizaciones.

\section{LISTA DE REFERENCIAS}

Aguinis, H., Villamor, I., Lazzarini, S., Vassolo, R., Amorós, J., \& Allen, D. (2020). Conducting Management Research in Latin America: Why and What's in It for You? Journal of Management, 45(5), 615-636. doi:10.1177/0149206320901581

Aiyappa, P., \& Mahesh, R. (2020). Elements influencing the small business. Test Engineering and Management, 83(5), 6785-6791. 
Boukamcha, F. (2019). The effect of transformational leadership on corporate entrepreneurship in Tunisian SMEs. Leadership and Organization Development Journal, 40(3), 286-304. doi:10.1108/LODJ-07-2018-0262

Chan, K., Ho, M., Chernyshenko, O., Bedford, O., Uy, M., Gomulya, D., . . Phan, W. (2012). Entrepreneurship, professionalism, leadership: A framework and measure for understanding boundaryless careers. Journal of Vocational Behavior, 81(1), 73-88.

Chang, Y., Chang, C., \& Chen, C. (2020). Transformational leadership and corporate entrepreneurship: Cross-level mediation moderation evidence. Leadership and Organization Development Journal, 38(6), 812-833. doi:10.1108/LODJ-10-20150219

Cogliser, C., \& Brigham, K. (2004). The intersection of leadership and entrepreneurship: Mutual lessons to be learned. Leadership Quarterly, 15(6), 771-799. doi:10.1016/j.leaqua.2004.09.004

Galloway, L., Kapasi, I., \& Sang, K. (2015). Entrepreneurship, Leadership, and the Value of Feminist Approaches to Understanding Them. Journal of Small Business Management, 53(3), 683-692. doi:10.1111/jsbm.12178

Leal, S. (2020). Las competencias blandas en los gerentes de proyecto de las organizaciones. Res Non Verba Revista Científica, 1-24. Obtenido de http://revistas.ecotec.edu.ec/index.php/rnv/article/view/286

Leal Paredes, S. Salomon, J., y Rivera Camino, J. (2021). Impact of Authentic Leadership on Work Engagement and Organizational Citizenship Behavior: The Meditation Role of Motivation for Work, International Journal of Economics \& Business Administration, 9(3), 3-31. DOI: 10.35808/ijeba/716

Cultural leadership ideals and social entrepreneurship: an international study. Journal of Social Entrepreneurship, 10(2), 108-128. doi:10.1080/19420676.2018.1541005

Lewis, K. (2015). Enacting Entrepreneurship and Leadership: A Longitudinal Exploration of Gendered Identity Work. Journal of Small Business Management, 53(3), 662-682. doi:10.1111/jsbm.12175

Morakinyo, A., \& Akinsola, O. (2019). Leadership and entrepreneurship education as a strategy for strengthening youth community engagement in Nigeria: Lessons learnt from jumpstart project. International Journal of Entrepreneurship, 23(1). 
Reid, S., Anglin, A., Baur, J., Short, J., \& Buckley, M. (2018). Blazing new trails or opportunity lost? Evaluating research at the intersection of leadership and entrepreneurship. Leadership Quarterly, 29(1), 150-164. doi:10.1016/j.leaqua.2017.11.005

Santos, J., Steil, A., \& Delgado-Hernández, D. (2020). State of the organizational learning field in Latin America and the Caribbean: Research methods and themes. Learning Organization, 27(2), 147-161. doi:10.1108/TLO-01-2019-0023

Sarabi, A., Froese, F., Chng, D., \& Meyer, K. (2020). Entrepreneurial leadership and MNE subsidiary performance: The moderating role of subsidiary context. $\begin{array}{llll}\text { International } & \text { Business } & \text { Review, } & 29(3),\end{array}$ doi:10.1016/j.ibusrev.2020.101672

Stephan, U., \& Pathak, S. (2016). Beyond cultural values? Cultural leadership ideals and entrepreneurship. Journal of Business Venturing, 31(5), 505-523. doi:10.1016/j.jbusvent.2016.07.003

Subramaniam, R., \& Shankar, R. (2020). Three Mindsets of Entrepreneurial Leaders. Journal of Entrepreneurship, 29(1), 7-37. doi:10.1177/0971355719893498

Vecchio, R. (2003). Entrepreneurship and leadership: Common trends and common threads. Human Resource Management Review, 13(2), 303-327. doi:10.1016/S1053-4822(03)00019-6

Zbierowski, P. (2020). Positive leadership and corporate entrepreneurship: Theoretical considerations and research propositions. Entrepreneurial Business and Economics Review, 4(3), 73-84. doi:10.15678/EBER.2016.040306 